\title{
Mesenchymal Glioblastoma
}

National Cancer Institute

\section{Source}

National Cancer Institute. Mesenchymal Glioblastoma. NCI Thesaurus. Code C111695.

A molecular subtype of glioblastoma characterized by the presence of NF1 mutations. 